\title{
Identification of IncRNA-mRNA regulatory network associated with isolated systolic hypertension and atherosclerotic cerebral infarction
}

\author{
Fang-Xiao Hu ${ }^{1 \#}$, Jie Yang ${ }^{1,2 \#}$, Chuan-Hua Yang ${ }^{2}$, Yan-Nan Tao ${ }^{3}$, Xue-Song Yang ${ }^{4}$, Ming-Ling Cui ${ }^{1}$, \\ Guan-Lan $\mathrm{Li}^{1}$, Chao $\mathrm{Li}^{5}$, Yue-Hua Jiang ${ }^{6}$
}

${ }^{1}$ Shandong University of Traditional Chinese Medicine, Jinan, China; ${ }^{2}$ Department of Cardiovascular, Affiliated Hospital of Shandong University of Traditional Chinese Medicine, Jinan, China; ${ }^{3}$ Shandong University of Traditional Chinese Medicine Second Affiliated Hospital, Jinan, China; ${ }^{4}$ Department of Vascular Surgery, Affiliated Hospital of Shandong University of Traditional Chinese Medicine, Jinan, China; ${ }^{5}$ Shandong University of Traditional Chinese Medicine, Jinan, China; ${ }^{6}$ Central Laboratory, Affiliated Hospital of Shandong University of Traditional Chinese Medicine, Jinan, China

Contributions: (I) Conception and design: FX Hu, J Yang; (II) Administrative support: CH Yang; (III) Provision of study materials or patients: FX Hu, J Yang, YN Tao, XS Yang, ML Cui, GL Li; (IV) Collection and assembly of data: FX Hu, J Yang, YN Tao, XS Yang, ML Cui, GL Li; (V) Data analysis and interpretation: FX Hu, J Yang, YN Tao, XS Yang, ML Cui, GL Li, C Li, YH Jiang; (VI) Manuscript writing: All authors; (VII) Final approval of manuscript: All authors.

"These authors contributed equally to this work and should be considered as co-first authors.

Correspondence to: Jie Yang; Chuan-Hua Yang. Department of Cardiovascular, Affiliated Hospital of Shandong University of Traditional Chinese Medicine, 16369 Jingshi Road, Jinan 250011, China. Email: sumuzheyangjie@163.com; yangchuanhua1962@126.com.

Background: Increasingly, evidence has shown that long non-coding RNAs (lncRNAs) play an important role in isolated systolic hypertension (ISH). However, a systematic lncRNA-messenger RNA (mRNA) regulatory network is still absent in isolated systolic hypertension and atherosclerotic cerebral infarction patients (ISH \& ACI).This research aimed to establish a lncRNA-mRNA co-expression network in patients with ISH \& ACI, to probe into the potential functions of lncRNA in such patients.

Methods: Expression profiles of lncRNA and mRNAs were collected and compared, from 8 patients with ISH and 8 patients with ISH \& ACI by RNA-seq data. Differentially expressed lncRNAs and mRNAs were screened out via high-throughput sequencing in the plasma of ISH/ACI patients and control ISH patients. Then, a lncRNA-mRNA interaction network was built using the Pearson correlation coefficient by Cytoscape software. The expression levels of the hub genes and lncRNAs were verified by quantitative reverse transcription-polymerase chain reaction (qRT-PCR) in another 10 ISH/ACI patients and 10 control patients. This study was approved by the responsible institutional review board (IRB) and informed consent was provided by participants.

Results: A total of 2,768 differentially expressed lncRNAs and 747 differentially expressed mRNAs were identified. We identified two hub genes (CD226 and PARVB) and 11 lncRNAs in the lncRNA-mRNA interaction network. The results of qRT-PCR and cell assay verified that lncRNAs ENST00000590604 and CD226 are highly expressed in patients of ISH \& ACI. Further, CD226 was associated with vascular endothelial cells growth and stability through the platelet activation and focal adhesion pathway.

Conclusions: We established a novel mRNA-lncRNA interaction network. The lncRNAs ENST00000590604 and CD226 might be the potential biomarkers of ISH \& ACI.

Keywords: Isolated systolic hypertension (ISH); atherosclerotic cerebral infarction (ACI); long non-coding RNA (lncRNA); messenger RNA (mRNA); heart and cerebral vessels

Submitted Sep 06, 2021. Accepted for publication Oct 20, 2021.

doi: 10.21037/atm-21-5176

View this article at: https://dx.doi.org/10.21037/atm-21-5176 


\section{Introduction}

Isolated systolic hypertension (ISH) is a major risk factor for cardiovascular morbidity and mortality, and the most common form of hypertension in the elderly population $(1,2)$. With an ageing population, the prevalence of hypertension, particularly ISH, is rapidly rising (2). Besides, ISH is associated with cerebrovascular diseases, such as isolated systolic hypertension and atherosclerotic cerebral infarction patients (ISH\&ACI) (2).

However, the underlying molecular mechanisms of ISH\&ACI have not been elucidated. Therefore, we sought the key molecules involved in ISH\&ACI and identified potential diagnostic biomarkers and therapeutic targets for ISH\&ACI.

Long non-coding RNAs (lncRNAs) are a class of lncRNA longer than 200 nucleotides. The lncRNAs are involved in many cellular processes, including chromatin remodeling, transcription modulation, and interacting with other RNAs to modulate gene expression (3). With the rapid technological advances in high-throughput sequencing, the study of lncRNAs has made remarkable progress (4). Accumulated experimental evidence shows that lncRNAs play an important role in the development of hypertension and might be the potential key regulators of hypertension $(5,6)$. The lncRNA TUG1/miR-145-5p/ FGF10 regulates the proliferation and migration of vascular smooth muscle cell in hypertension by activating the Wnt/ $\beta$-catenin pathway (6). Down-regulation of MRAK048635_ P1 is probably an important factor for vascular remodeling in essential hypertension (7). Nevertheless, the relationship between lncRNAs and ISH has not yet been reported.

In this paper, 946 up-regulated and 1,822 downregulated differentially expressed lncRNAs, and 288 upregulated and 519 down-regulated differentially expressed mRNAs were identified between ISH\&ACI cases and ISH controls, respectively. The Gene Ontology (GO) and Kyoto Encyclopedia of Genes and Genomes (KEGG) enrichment analyses revealed that differentially expressed lncRNAs and mRNAs were significantly enriched in platelet activation. According to the lncRNA-mRNA co-expression network, two hub genes (CD226 and $P A R V B)$ were identified and 9 lncRNAs were predicted to target these two hub genes. Then, the lncRNA-mRNA pairs were verified by real-time polymerase chain reaction (qPCR) in 20 individual samples. Besides, flow cytometer (FCM) was performed to evaluate proliferation and apoptosis rate and $\mathrm{qPCR}$ was performed to validate the lncRNA-mRNA pairs in human brain microvascular endothelial cells (HBMECs).

In summary, our results showed that lncRNAs ENST00000590604 regulated the expression of CD226 through platelet activation and focal adhesion pathway. The lncRNAs ENST00000590604 and CD226 might be the potential biomarkers of ISH and atherosclerotic cerebral infarction patients.

We present the following article in accordance with the MDAR reporting checklist (available at https://dx.doi. org/10.21037/atm-21-5176).

\section{Methods}

\section{Study samples}

A total of 18 ISH\&ACI patients were recruited from Affiliated Hospital of Shandong University of Traditional Chinese Medicine from May 2019 to April 2020. We defined ISH and ACI according to the 2018 revised edition of the Chinese guidelines for the prevention and treatment of hypertension (exposure draft) and 2015 China Cerebrovascular Disease Classification. Meanwhile, patients conforming to the following standards were included: (I) aged 18 years or above, no gender limitations; (II) within 2 weeks to 6 months after the occurrence of cerebral infarction; (III) willing to participate in this experiment, cooperate with the doctors, and sign the informed consent in writing. A total of 18 ISH controls were recruited from the same hospital during the same period. We randomly selected 8 ISH\&ACI patients and 8 ISH controls from 18 ISH\&ACI patients and 18 ISH controls for RNA sequencing, and the remaining samples were used as replication population for the validation of differentially expressed lncRNAs and mRNAs. Among the participants selected this time, 21 were male and 15 were female. All participants were aged about 50-90 years.

The study was approved by the Ethics Committee of Affiliated Hospital of Shandong University of Traditional Chinese Medicine (ethical review approval number: AF/ SC-08/03.0). Written informed consent was provided by all participants or their families under the Declaration of Helsinki (as revised in 2013).

\section{$R N A$ extraction, library preparation, and $R N A-s e q$}

We extracted RNA from plasma using the QIAamp Circulating Nucleic Acid Kit (QIAGEN, Hilden, Germany) according to the manufacturer's protocol. The RNA 
was then purified and concentrated using the RNeasy MinElute Cleanup Kit (QIAGEN, Germany). The quality of RNA was evaluated using NanoDrop 2000 (Thermo Fisher, Waltham, MA, USA). Sequencing libraries were generated using TruSeq Stranded Total RNA LT - (with Ribo - Zero TM Gold) - Set A (Illumina, San Diego, CA, USA) following the manufacturer's recommendations. The libraries were then sequenced on the Illumina Hiseq 2500 platform and $125 \mathrm{bp} / 150 \mathrm{bp}$ paired-end reads were generated.

\section{Quantitative real-time PCR validation}

Quantitative real-time PCR (qRT-PCR) was used to validate selected lncRNAs and mRNAs in 20 individual samples. Reverse transcription was performed using the PrimeScript ${ }^{\mathrm{TM}}$ RT reagent Kit with gDNA Eraser (SparkJade Scientific Instruments Co., Ltd., Shandong, China). Then, qRT-PCR was performed using a TB Green ${ }^{\circledR}$ Premix Ex Taq ${ }^{\mathrm{TM}}$ II (SparkJade, Shandong, China) and an Applied Biosystems 7500 Real-Time PCR System (Thermo Fisher, USA) according to standard methods. Specific primers of lncRNAs and mRNAs are listed in Table S1. The $2^{-\Delta \Delta C T}$ method was used to calculate the relative fold-change to the house-keeping gene ( $\beta$-action).

\section{Cell experiments}

\section{Cell culture}

The HBMECs (Hunan Fenghui Biotechnology Co. Ltd., Hunan, China, Cat.CL0116) were cultured with high glucose $(4.5 \mathrm{mg} / \mathrm{mL})$ Dulbecco's modified Eagle medium (DMEM; Gibco, Amarillo, TX, USA, REF.\#C11995065BT) and supplemented with $10 \%$ fetal bovine serum (FBS; Gibco, REF.10099141) and 1\% penicillin/streptomycin solution (P/S; HyClone, Logan, UT, USA, Cat. \#SV30010) at $37{ }^{\circ} \mathrm{C}$ in a humidified $5 \% \mathrm{CO}_{2}$ incubator. Then, HBMECs were intervened by sodium pyruvate solution Ang II (Solarbio Co., Ltd., Beijing, China) and oxygenglucose deprivation/reoxygenation (OGD/R). We used HBMECs exposed to Ang II and normoxia as controls.

\section{3-(4,5-dimethylthiazol-2-yl)-2,5-diphenyl tetrazolium bromide assay}

The effect of Ang II and OGD/R on proliferation were detected by 3-(4,5-dimethylthiazol-2-yl)-2,5-diphenyl tetrazolium bromide assay (MTT) assay. The HBMECs were seeded at a density of 1,500 cells per well into 96-well microplates in a final volume of $100 \mu \mathrm{L}$ with $2 \times 10^{-6} \mathrm{~mol} / \mathrm{L}$ of Ang II, and they were maintained for 12, 24, 48, or $72 \mathrm{~h}$ at $37{ }^{\circ} \mathrm{C}$ in a humidified atmosphere containing $5 \% \mathrm{CO}_{2}$. After that, HBMECs were cultured in Earle's Balanced Salt Solution (EBSS, Solarbio, Cat.H2025) without glucose and FBS and deprived of oxygen by transferring into an anaerobic incubator $\left(0 \% \mathrm{O}_{2} / 5 \% \mathrm{CO}_{2} / 95 \% \mathrm{~N}_{2}\right)$ for $4 \mathrm{~h}$. After OGD, cells were returned to a normoxic incubator under $5 \% \mathrm{CO}_{2} / 95 \%$ air for $24 \mathrm{~h}$ in complete medium. Subsequently, to monitor cell viability, $10 \mu \mathrm{L}$ of sterile MTT (Solarbio, Lot.\#303H0525, Beijing, China) at a final concentration of $5 \mathrm{mg} / \mathrm{mL}$ was added to each well for incubation at $37^{\circ} \mathrm{C}$ for $4 \mathrm{~h}$; this was followed by the administration of $150 \mu \mathrm{L}$ dimethyl sulfoxide solution (DMSO; Sinopharm Chemical Reagent Co., Ltd., Shanghai, China, Cat. \#30072418, Shanghai, China) per well to dissolve the formazan crystals. The optical density (OD) value was measured at $562 \mathrm{~nm}$ using a Multiskan GO microplate reader (Thermo Fisher Scientific, Waltham, MA, USA) according to the manufacturer's instruction. The OD-value indicated the counts of living cells.

\section{Apoptosis rate assay}

The effect of Ang II and OGD/R on apoptosis rate was assessed using Annexin V staining by Muse Cell Analyzer (Merck, Darmstadt, Germany Millipore, Burlington, MA, USA), according to the manufacturer's directions.

\section{qRT-PCR}

To validate the sequence data, 11 differentially expressed genes(DEGs) related to endothelial dysfunction were chosen for qRT-PCR, including 8 lncRNAs (ENST00000565493, ENST00000606054, ENST00000590604,NONHS AT138949.2, ENST00000566942, ENST00000369385, NONHSAT2 17189.1, ENST00000540082) and their corresponding target mRNAs (CD226, PARVB). After being cultured in 6-well plates at a density of 6,000 cells $/ \mathrm{cm}^{2}$, total RNAs from model cells and control cells were extracted using SPARKeasy Improved Tissue/Cell RNA Kit (Haosail Science Co., Shandong, China, Lot.YADKZ). PrimeScript ${ }^{\mathrm{TM}} \mathrm{RT}$ reagent Kit with gDNA Eraser (Perfect Real Time) (TaKaRa, Cat.\#RR047A) and reverse transcription reaction according to the manufacturer's standard operation process was used to synthesize the DNA template. TB Green ${ }^{\text {TM }}$ Premix Ex Taq ${ }^{\text {TM }}$ II (Tli RNaseH Plus) (TaKaRa, Kusatsu, Shiga, Japan, Cat.\#RR820A) was used to mplify qRT-PCR following the manufacturer's instructions. The expression of lncRNA was normalized 
to glyceraldehyde 3-phosphate dehydrogenase (GAPDH) as described in the literature. Complementary DNA (cDNA) was synthesized using a Transcriptor First Strand cDNA Synthesis Kit (Roche, Mannheim, Germany, Cat. \#04379012001), and qRT-PCR was performed by using LightCycler 480 SYBR Green I Master (Roche, Cat. \#04707516001), according to the manufacturer's instructions, using a LightCycler480 instrument (Roche). The primer sequences are listed in Table S1 (Sangon Biotech, Shanghai, China). The data were calculated and presented by the $2^{-\Delta \Delta C T}$ method.

\section{Statistical analysis}

All statistical analyses were performed using the R 3.6.0 (http://www.R-project.iorg/). The two-tailed Student's $t$-test was used for normally distributed variables and the Wilcoxon Rank-Sum test was used for abnormally distributed data. Data normality was evaluated using the Shapiro-Wilk test. Differences between the case and control group were considered significant at $\mathrm{P}$ value $<0.05$.

\section{Results}

\section{Identification of DEGs and differentially expressed IncRNAs}

Read counts obtained from RNA-seq were normalized as fragments per kilobase of transcript per million mapped fragments (FPKM) (8). The FPKM was used to identify the differentially expressed lncRNAs (DELs) and differentially expressed mRNAs in ISH\&ACI cases $(n=8)$ and ISH controls $(n=8)$. The DELs and differentially expressed mRNAs met the following cutoff criteria: $\mathrm{P}$ value $<0.05$ and I FoldChange I >2 (9). A total of 2,768 DELs (946 up-regulated and 1,822 down-regulated) and 747 differentially expressed mRNAs (288 up-regulated and 519 down-regulated) were identified between ISH\&ACI cases and ISH controls. Volcano plots were used to visualize the distribution of the DELs (Figure 1A) and differentially expressed mRNAs (Figure 1B). The hierarchical clustering plots showed clear separation and consistency in the expression profiles of ISH\&ACI cases and ISH controls (Figure 1C,1D).

\section{GO and KEGG patbway analysis}

We performed GO and KEGG enrichment analyses by hypergeometric test to find significantly enriched GO terms and KEGG pathways of DELs and differentially expressed mRNAs. By GO enrichment analysis, DELs and differentially expressed mRNAs were classified into three categories: cellular component (CC), molecular function (MF), and biological process (BP). The potential mRNAs targets of DELs were predicted using cis-specific binding modules and trans-specific binding modules. The intersection of targets of DELs and differentially expressed $m R N A s$ were used to perform enrichment analysis.

As shown in Figure $2 A$ and Table S2, GO enrichment analysis identified 42 significant $\mathrm{GO}$ terms ( $\mathrm{P}$ value $\leq 0.05$ ). In BP, the top 4 GO terms were positive regulation of blood coagulation, positive regulation of coagulation, cellular response to glucagon stimulus, and platelet degranulation; in $\mathrm{CC}$, the top $4 \mathrm{GO}$ terms were platelet alpha granule, platelet alpha granule lumen, secretory granule lumen, and cytoplasmic membrane-bounded vesicle lumen; in $\mathrm{MF}$, the top $2 \mathrm{GO}$ terms were integrin binding and cell adhesion molecule binding. The KEGG enrichment analysis identified 6 significant KEGG pathways (Figure $2 B$ and Table S3). The 6 significant KEGG pathways were arrhythmogenic right ventricular cardiomyopathy, platelet activation, extracellular matrix (ECM)-receptor interaction, hypertrophic cardiomyopathy, dilated cardiomyopathy, and focal adhesion. The enrichment analyses showed that the intersection of targets of DELs and differentially expressed mRNAs were mainly associated with platelet activation.

\section{LncRNA-mRNA co-expression network construction and bub genes analysis}

We calculated the Pearson correlation coefficient using the expression value of lncRNAs and mRNAs. We identified lncRNA-mRNA co-expression with coefficient great than 0.9 and the $\mathrm{P}$ value less than 0.05 . According to the results of IncRNA-mRNA co-expression, we then constructed the lncRNA-mRNA network using Cytoscape software (https:// cytoscape.org/). We identified 2 hub genes in the network, $C D 226$ and PARVB (Figure 3).

The hub gene CD226 (also known as DNAM-1, PTA-1, or TLiSA-1) is an immunoglobulin-like glycoprotein. It was shown to be expressed on the platelet membrane and involved in platelet activation $(10,11)$. Platelets play a crucial role in the control of acute hemorrhage and thrombosis, and thus are involved in atherosclerosis and myocardial infarction (12). It may be that $C D 226$ participates in atherosclerotic cerebral infarction. The other hub gene, $P A R V B$ (also known as beta-parvin and affixin) is a member of the parvin family of 
A

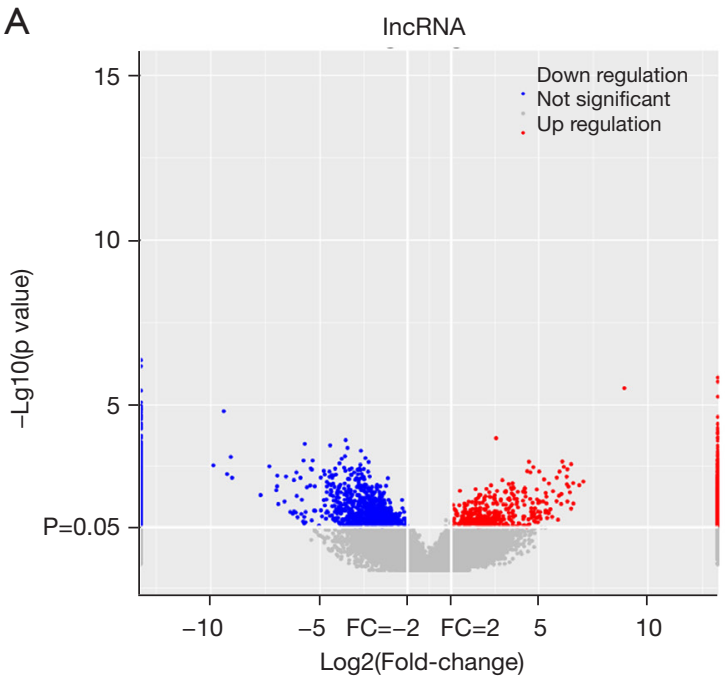

C

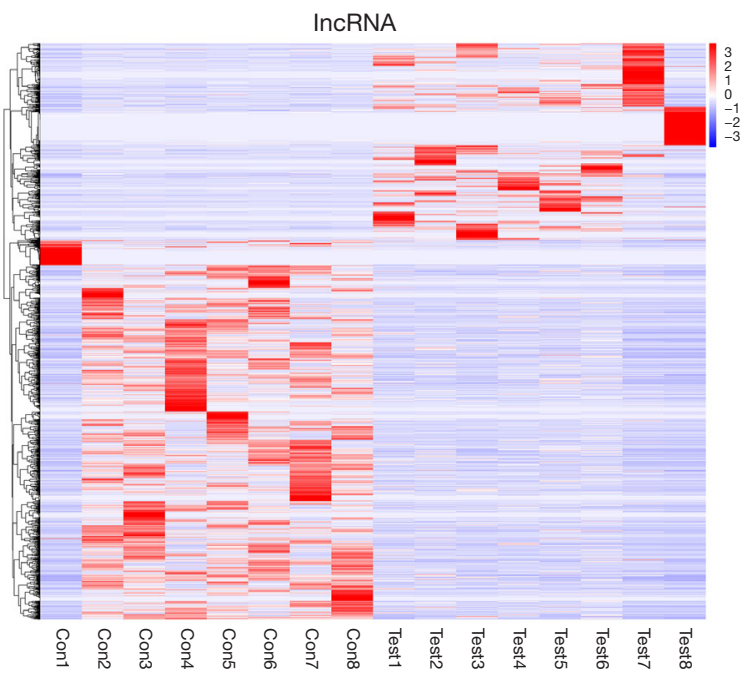

B

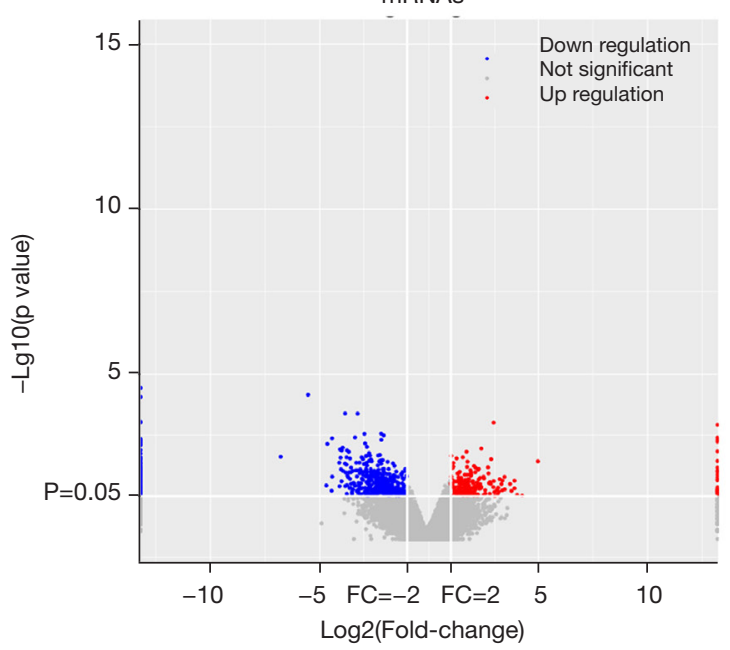

D

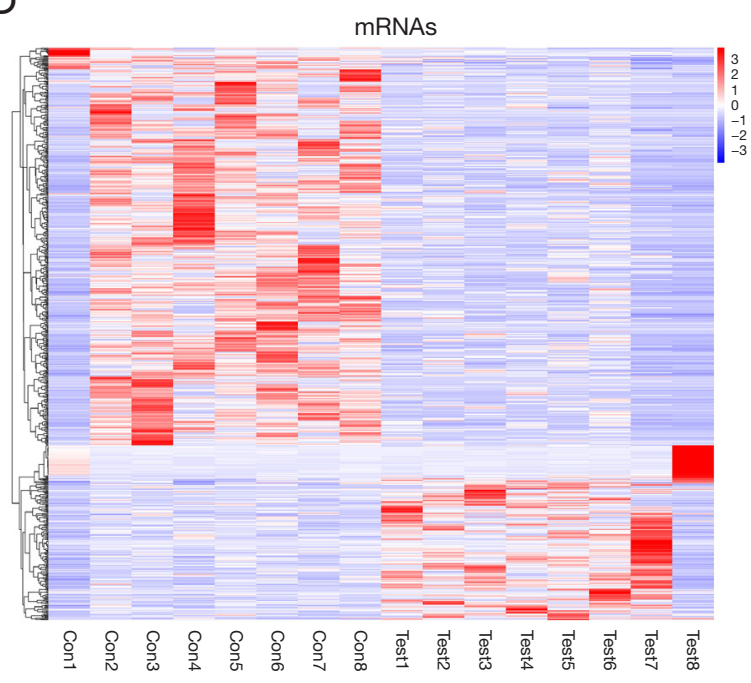

Figure 1 Differentially expressed lncRNAs and mRNAs in ISH\&ACI cases $(n=8)$ and ISH controls $(n=8)$. (A) Volcano plots of differentially expressed lncRNAs. The $\mathrm{X}$-axis is $\log 2$ Fold-Change and the $\mathrm{Y}$-axis is $\log 10 \mathrm{p}$-value. Red (up-regulated) and blue (down-regulated) points indicated the differential expression lncRNA; (B) Volcano plots of differentially expressed mRNAs; (C) differentially expressed lncRNAs hierarchical clustering, each column represents a sample and every row represents a lncRNA. Red indicates high relative expression and blue indicates low relative expression; (D) differentially expressed mRNAs hierarchical clustering. ISH\&ACI, isolated systolic hypertension and atherosclerotic cerebral infarction; DEGs, differentially expressed genes; DELs, differentially expressed lncRNAs; lncRNAs, long noncoding RNAs; mRNAs, messenger RNAs.

actin-binding proteins, which plays a role in cytoskeleton organization and cell adhesion (13). It was shown to be associated with coronary restenosis, hematoma volume, and spontaneous intracerebral hemorrhage $(14,15)$.

\section{The validation of expression profiles of $\ln \mathrm{R} R A \mathrm{~s}$}

We performed qRT-PCR for further validation of 9 selected differentially expressed lncRNA-mRNA pairs in 20 individual samples (Table 1). We found that the expression of CD226 and lncRNAs ENST00000590604 were significantly up-regulated in the cases compared to controls (Figure 4). The results of lncRNAs ENST00000590604 and CD226 were consistent with the results of RNA-seq data. The hub gene $C D 226$ was significantly upregulated in ISH\&ACI patients. 


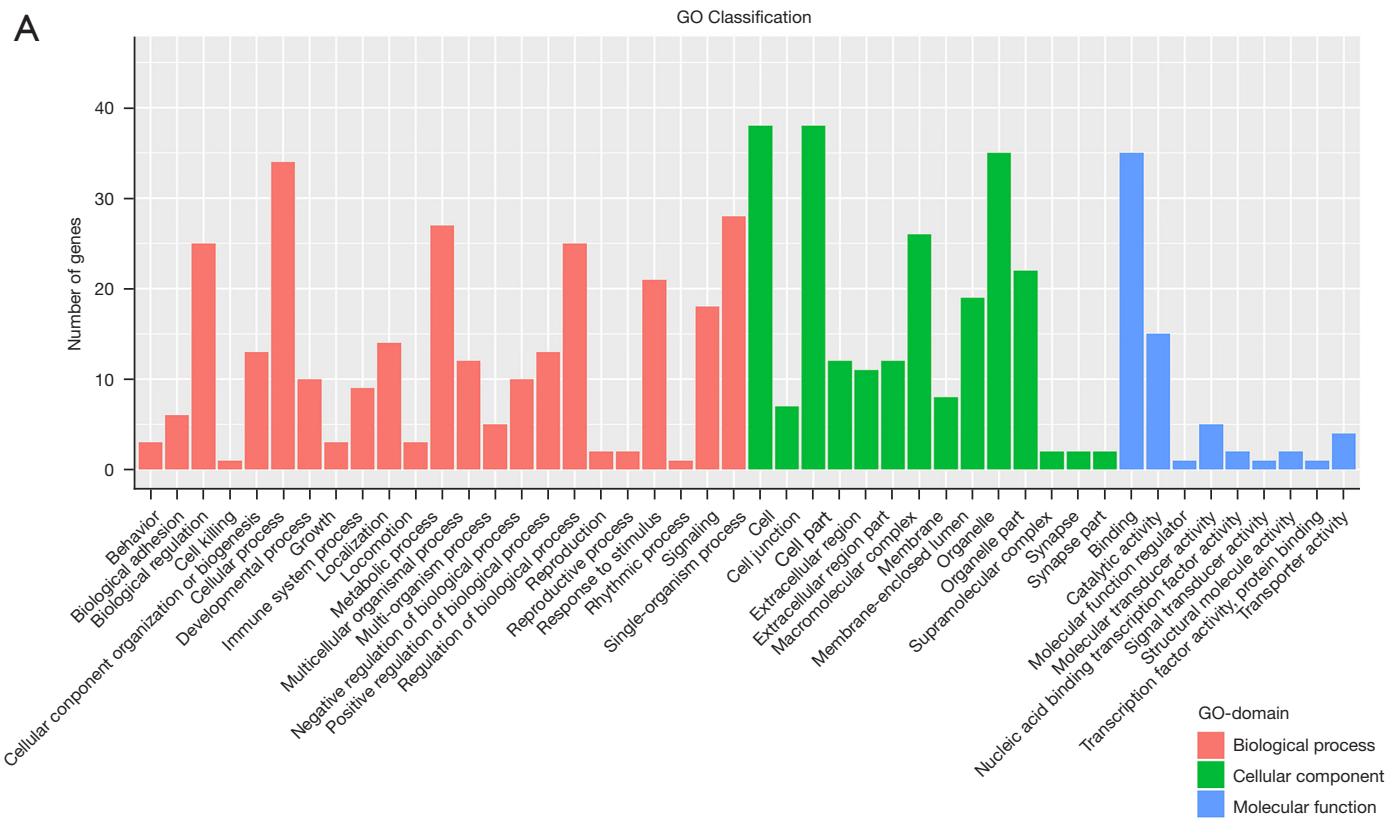

B

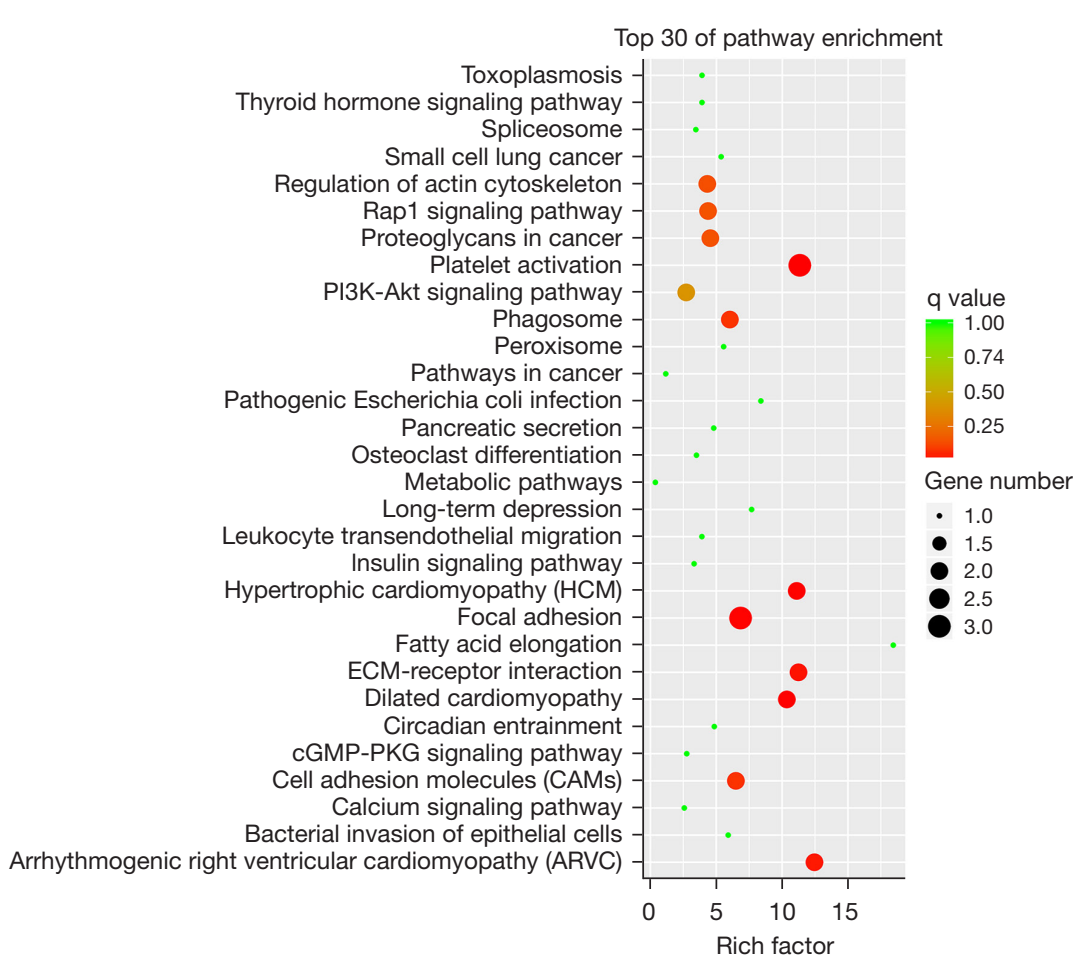

Figure 2 GO and KEGG pathway enrichment analysis. (A) GO enrichment contains 3 terms: cellular component, molecular function, and biological process; (B) the top 30 enriched KEGG pathways. GO, Gene Ontology; KEGG, Kyoto Encyclopedia of Genes and Genomes. 

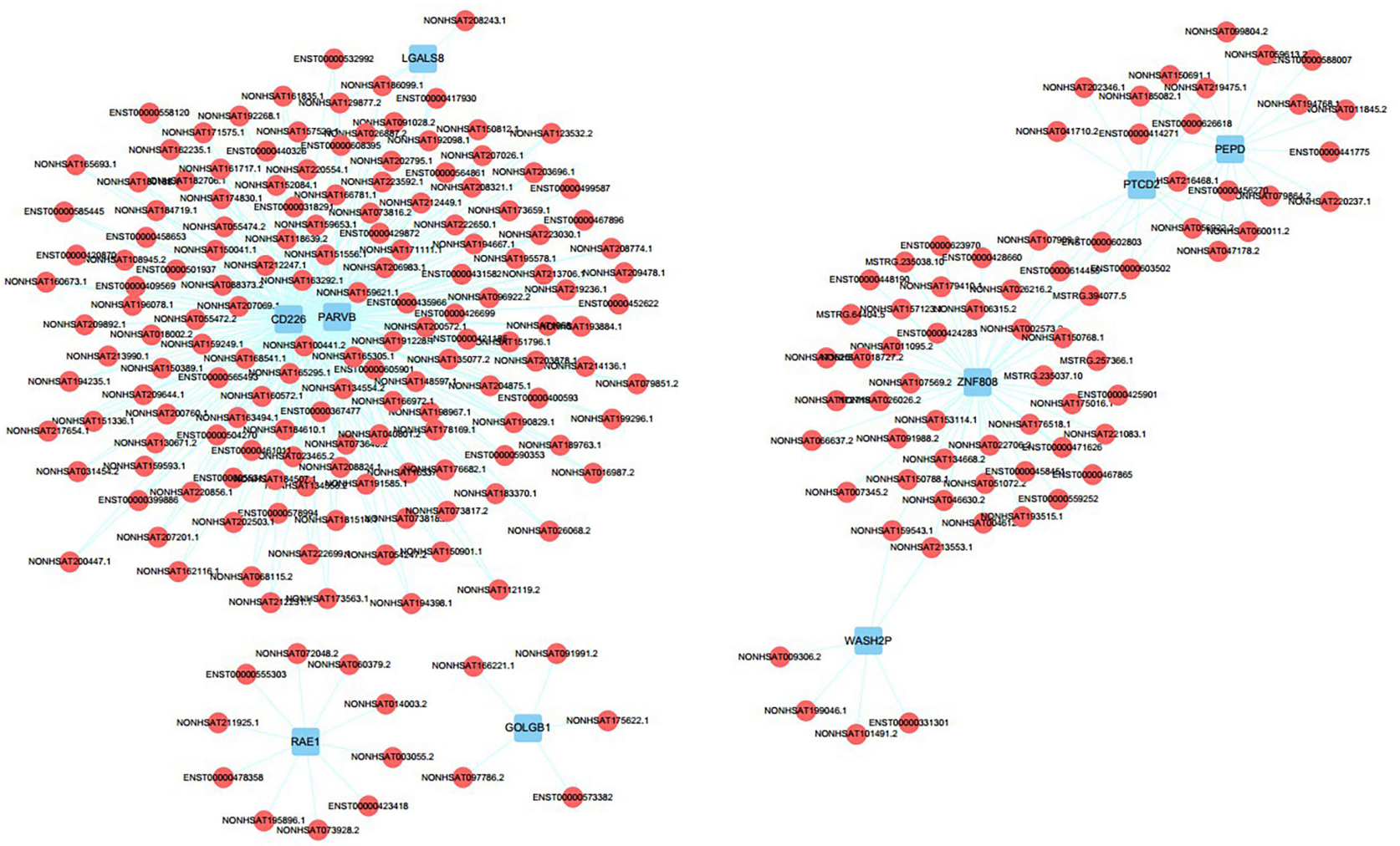

Figure 3 LncRNA-mRNA co-expression network. Red indicates lncRNAs and blue indicates mRNAs. The top 2 hub genes are CD226 and PARVB. lncRNAs, long non-coding RNAs; mRNAs, messenger RNAs.

Table 1 Differentially expressed lncRNA-mRNA pairs

\begin{tabular}{ll}
\hline Target & IncRNAs \\
\hline CD226 & ENST00000565493, ENST00000606054, ENST00000590604, NONHSAT217189.1, ENST00000566942 \\
PARVB & ENST00000565493, NONHSAT138949.2, ENST00000369385, ENST00000540082 \\
\hline
\end{tabular}

\section{The effects of Ang II and OGD/R on HBMECs}

We performed MTT assay and FCM to evaluate proliferation and apoptosis rate. The results indicated that, compared with the control group, HBMECs' growth was inhibited and apoptosis rate was increased, which were treated with OGD/ $\mathrm{R}$ (Figure 5).

\section{qRT-PCR validation of significant DELs and differentially expressed mRNAs of HBMECs}

The qRT-PCR results using HBMECs were consistent with the RNA-Seq results using plasma of patients, including lncRNA ENST00000590604 and CD226 (Figure 6).

\section{Discussion}

\section{Summary}

Hypertension has become one of the most important risk factors for cardiovascular and cerebrovascular accidents in the world today and contributes to an elevated risk of stroke, myocardial infarction, heart failure, and renal failure $(16,17)$. Globally, hypertension affects $31.1 \%$ of adults (18). The cost of hypertension has an enormous public health burden. In elderly men and young adults, ISH is the most common type of hypertension (19).

The emerging evidence indicates that IncRNAs play a critical role in various biological functions and disease processes (20). For example, HOTAIR (HOX transcript 

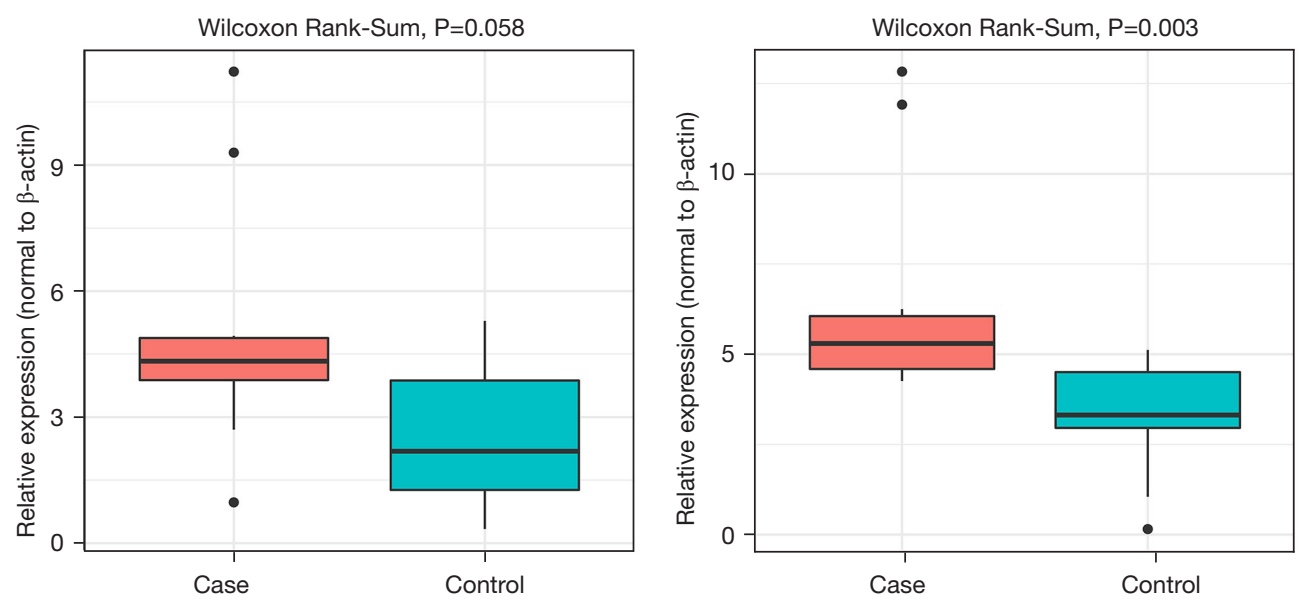

Figure 4 Relative levels of mRNAs and lncRNAs in 10 cases and 10 controls. Using qRT-PCR to calculate the relative fold-change to the house-keeping gene ( $\beta$-action). From left to right are lncRNA ENST00000590604 and CD226. mRNA, messenger RNA; lncRNA, long non-coding RNA; qRT-PCR, quantitative reverse transcription-polymerase chain reaction.

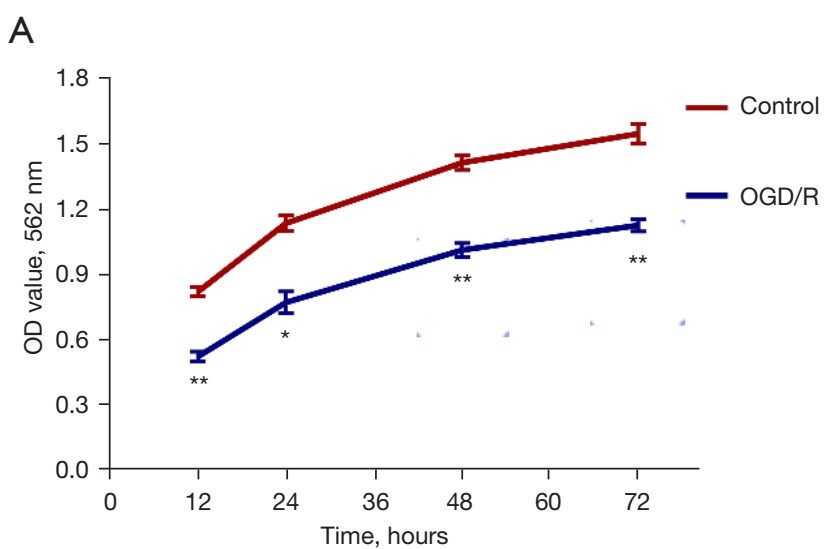

C

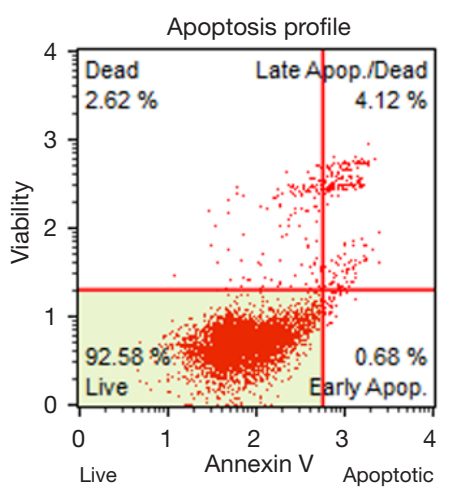

B

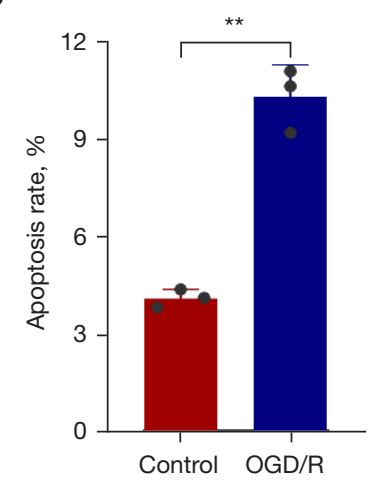

D

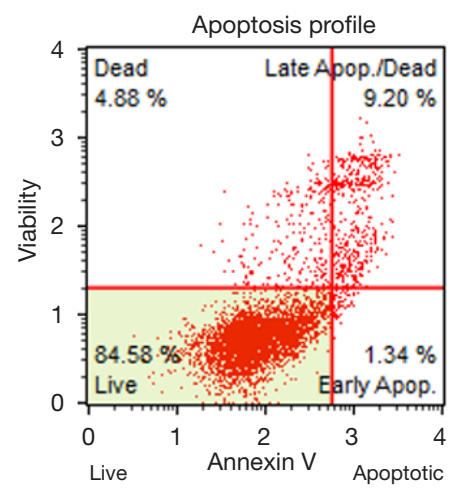

Figure 5 The effect of OGD/R on proliferation and apoptosis rate of HBMECs. (A) HBMECs' viability was declined after OGD/R compared with control group $\left({ }^{*} \mathrm{P}<0.05,{ }^{* *} \mathrm{P}<0.01\right.$ vs. Control group). (B) The apoptosis rate of $\mathrm{OGD} / \mathrm{R}$ group was increased compared with control group ${ }^{* *} \mathrm{P}<0.01$ vs. Control group). (C) Control group apoptosis results. (D) OGD/R group apoptosis results. OGD/R, oxygenglucose deprivation/reoxygenation; HBMECs, human brain microvascular endothelial cells. 


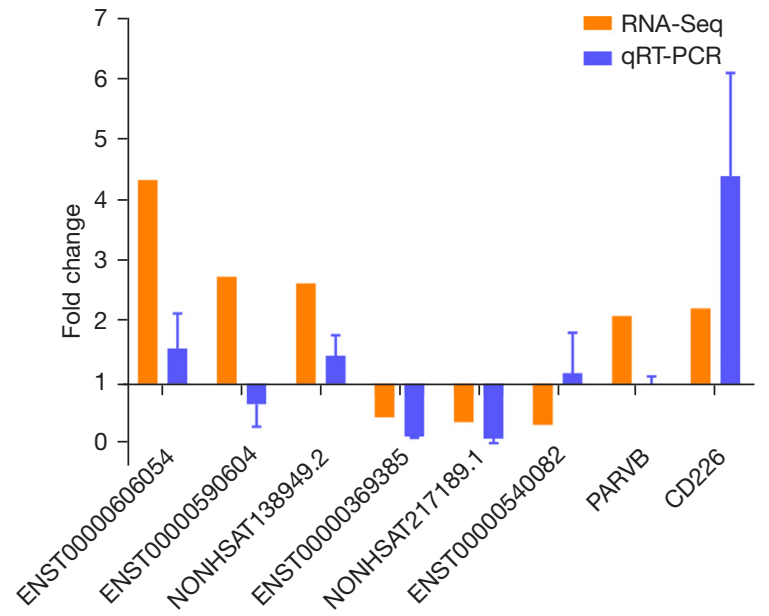

Figure 6 qRT-PCR validation $(\mathrm{n}=10)$ of DEGs compared to RNA-Seq $(n=8)$. Yellow represents the expression of DELs and DE mRNAs in the ISH complicated with stroke group versus the ISH group by RNA-Seq, blue represents the expression of DELs and DE mRNAs in the Ang II and OGD/R group versus the control group by qRT-PCR. qRT-PCR, quantitative reverse transcriptionpolymerase chain reaction; DEGs, differentially expressed genes; DELs, differentially expressed long non-coding RNAs; DE, differentially expressed; ISH, isolated systolic hypertension; OGD/ $\mathrm{R}$, oxygen-glucose deprivation/reoxygenation.

antisense intergenic RNA) is one of the most well-studied lncRNAs that are overexpressed in a variety of cancers (21). Though the significance of lncRNAs is being increasingly found in hypertension, the expression profile and the mechanism of lncRNAs remain unclear in ISH.

In this study, our RNA-seq data revealed multiple lncRNAs dysregulated in ISH\&ACI. The GO and KEGG enrichment analyses found that the intersection of the targets of DEls and DE mRNAs were mainly associated with platelet activation, ECM-receptor interaction, and focal adhesion. Platelet activation signaling pathway plays a critical role in the function of platelets in hemostasis and thrombosis (22). Activated platelets aggregate at the site of endothelial cell erosion, stimulating thrombus formation and promoting atherothrombotic disease (23). The focal adhesion signaling pathway promotes cell migration and angiogenesis $(24,25)$.

We also found 2 upregulated hub genes in ISH\&ACI (CD226 and PARVB) by the lncRNA-mRNA co-expression network. The gene CD226 is an immunoglobulin-like glycoprotein and mediates platelet and megakaryocytic cell adhesion to vascular endothelial cells $(11,26)$. Research has suggested that platelet-derived miR-142-3p regulating apoptosis of endothelial cells in hypertension and the dysfunction of vascular endothelial cells play crucial roles in vascular remodeling during hypertension $(27,28)$. The $P A R V B$ gene has been found to affect human endothelial cell shape and motility, suggesting an important role of $P A R V B$ in vessel growth and vessel stability $(29,30)$.

We further validated the results by qRT-PCR in another group of 20 patients. FCM was also performed to evaluate proliferation and apoptosis rate in HBMECs cells and real-time PCR was performed to validate the lncRNAmRNA pairs in HBMECs cells. Here, we found lncRNAs ST00000590604 regulated the expression of CD226. The CD226 gene was associated with vascular endothelial cells growth and stability through platelet activation and focal adhesion pathway. The lncRNAs ENST00000590604 and CD226 could be the potential biomarkers for ISH\&ACI and this finding provides a new idea for the clinical treatment of ISH\&ACI.

\section{Limitations}

There were several limitations to this study, as follows: the cases included in this research were limited in number, thus a larger sample size is necessary for future research to generate more reliable results. Also, since no medication targeted the differentially expressed lncRNAs or mRNAs, further research on the pharmacological mechanism is still needed to unveil the essential roles of these potential biomarkers. This study did not explore the relative expression of lncRNA associated with disease risk, severity, and inflammatory cytokine levels in patients with systolic hypertension with atherosclerotic cerebral infarction. No animal experiments were performed to verify the results. This paper did not explore the relationship between polymorphisms of lncRNA gene and isolated systolic hypertension and atherosclerotic cerebral infarction. There is no condition to add co-expression module analysis.

\section{Comparison with existing literature}

Past research has mostly focused on the differential expressions of lncRNAs and mRNAs in ACI patients and healthy controls or in hypertension patients and healthy controls. The differential expressions of lncRNAs in ISH\&ACI patients and ISH patients have not been investigated. We initiated the probe into the differentially 
expressed lncRNAs and mRNAs in ISH\&ACI patients and ISH patients at the transcriptive level in hope of demonstrating the potential mechanism for the progression of the disease.

\section{Implications for research and practice}

The findings of this study allow discussion of the physiological foundation for ISH\&ACI from the level of lncRNAs via high-throughput sequencing. We drew a preliminary conclusion on the potential pathomechanism for ISH patients to lapse into ACI. Our research provides a theoretical foundation for future precise diagnoses and treatments with medication.

\section{Conclusions}

We have established a novel mRNA-lncRNA interaction network. The lncRNAs ENST00000590604 and CD226 might be the potential biomarkers of ISH\&ACI. This exploratory work generates a novel mRNA-lncRNA interaction network that was never before been carried out in ISH\&ACI patients. The two lncRNAs ENST00000590604 and $C D 226$ verified as potential biomarkers for ISH\&ACI had not been mentioned in former literature.

\section{Acknowledgments}

Funding: This work was supported by the National Natural Science Foundation of China (81804061); Shandong Province 'Taishan Scholar' Construction Project Funds (2018-35); Ji'nan Science and Technology Project (201805078).

\section{Footnote}

Reporting Checklist: The authors have completed the MDAR reporting checklist. Available at https://dx.doi. org/10.21037/atm-21-5176

Data Sharing Statement: Available at https://dx.doi. org/10.21037/atm-21-5176

Conflicts of Interest: All authors have completed the ICMJE uniform disclosure form (available at https://dx.doi. org/10.21037/atm-21-5176). The authors have no conflicts of interest to declare.

Ethical Statement: The authors are accountable for all aspects of the work in ensuring that questions related to the accuracy or integrity of any part of the work are appropriately investigated and resolved. The study was approved by the Ethics Committee of Affiliated Hospital of Shandong University of Traditional Chinese Medicine (ethical review approval number: AF/SC-08/03.0). Written informed consent was provided by all participants or their families under the Declaration of Helsinki (as revised in 2013).

Open Access Statement: This is an Open Access article distributed in accordance with the Creative Commons Attribution-NonCommercial-NoDerivs 4.0 International License (CC BY-NC-ND 4.0), which permits the noncommercial replication and distribution of the article with the strict proviso that no changes or edits are made and the original work is properly cited (including links to both the formal publication through the relevant DOI and the license). See: https://creativecommons.org/licenses/by-nc-nd/4.0/.

\section{References}

1. Mancia G, Giannattasio C. Diagnostic and therapeutic problems of isolated systolic hypertension. J Hypertens 2015;33:33-43.

2. Bavishi C, Goel S, Messerli FH. Isolated Systolic Hypertension: An Update After SPRINT. Am J Med 2016;129:1251-8.

3. Lorenzen JM, Thum T. Long noncoding RNAs in kidney and cardiovascular diseases. Nat Rev Nephrol 2016;12:360-73.

4. Jathar S, Kumar V, Srivastava J, et al. Technological Developments in lncRNA Biology. Adv Exp Med Biol 2017;1008:283-323.

5. Jusic A, Devaux Y; EU-CardioRNA COST Action (CA17129). Noncoding RNAs in Hypertension. Hypertension 2019;74:477-92.

6. Shi L, Tian C, Sun L, et al. The lncRNA TUG1/miR$145-5 \mathrm{p} / \mathrm{FGF} 10$ regulates proliferation and migration in VSMCs of hypertension. Biochem Biophys Res Commun 2018;501:688-95.

7. Fang G, Qi J, Huang L, et al. LncRNA MRAK048635_ P1 is critical for vascular smooth muscle cell function and phenotypic switching in essential hypertension. Biosci Rep 2019;39:BSR20182229.

8. Mortazavi A, Williams BA, McCue K, et al. Mapping and quantifying mammalian transcriptomes by RNA-Seq. Nat Methods 2008;5:621-8.

9. Zhao X, Tang DY, Zuo X, et al. Identification of lncRNA- 
miRNA-mRNA regulatory network associated with epithelial ovarian cancer cisplatin-resistant. J Cell Physiol 2019;234:19886-94.

10. Scott JL, Dunn SM, Jin B, et al. Characterization of a novel membrane glycoprotein involved in platelet activation. J Biol Chem 1989;264:13475-82.

11. Kojima H, Kanada H, Shimizu S, et al. CD226 mediates platelet and megakaryocytic cell adhesion to vascular endothelial cells. J Biol Chem 2003;278:36748-53.

12. Zhang J, Ding Y, Jiang D, et al. Deficiency of platelet adhesion molecule CD226 causes megakaryocyte development and platelet hyperactivity. FASEB J 2020;34:6871-87.

13. Yamaji S, Suzuki A, Sugiyama Y, et al. A novel integrinlinked kinase-binding protein, affixin, is involved in the early stage of cell-substrate interaction. J Cell Biol 2001;153:1251-64.

14. Marini S, Devan WJ, Radmanesh F, et al. 17p12 Influences Hematoma Volume and Outcome in Spontaneous Intracerebral Hemorrhage. Stroke 2018;49:1618-25.

15. Verschuren JJ, Trompet S, Sampietro ML, et al. Pathway analysis using genome-wide association study data for coronary restenosis--a potential role for the PARVB gene. PLoS One 2013;8:e70676.

16. Lawes CM, Vander Hoorn S, Rodgers A, et al. Global burden of blood-pressure-related disease, 2001. Lancet 2008;371:1513-8.

17. Li W, Gu H, Teo KK, et al. Hypertension prevalence, awareness, treatment, and control in 115 rural and urban communities involving 47000 people from China. J Hypertens 2016;34:39-46.

18. Mills KT, Bundy JD, Kelly TN, et al. Global Disparities of Hypertension Prevalence and Control: A Systematic Analysis of Population-Based Studies From 90 Countries. Circulation 2016;134:441-50.

19. Kobalava ZD, Kotovskaya YV. Isolated Systolic Hypertension in Different Ages. Kardiologiia

Cite this article as: $\mathrm{Hu} \mathrm{FX}$, Yang J, Yang $\mathrm{CH}$, Tao YN, Yang XS, Cui ML, Li GL, Li C, Jiang YH. Identification of lncRNA-mRNA regulatory network associated with isolated systolic hypertension and atherosclerotic cerebral infarction. Ann Transl Med 2021;9(20):1589. doi: 10.21037/atm-21-5176
2015;5 5:84-90

20. Peng WX, Koirala P, Mo YY. LncRNA-mediated regulation of cell signaling in cancer. Oncogene 2017;36:5661-7.

21. Bhan A, Soleimani M, Mandal SS. Long Noncoding RNA and Cancer: A New Paradigm. Cancer Res 2017;77:3965-81.

22. Estevez B, Du X. New Concepts and Mechanisms of Platelet Activation Signaling. Physiology (Bethesda) 2017;32:162-77.

23. Yun SH, Sim EH, Goh RY, et al. Platelet Activation: The Mechanisms and Potential Biomarkers. Biomed Res Int 2016;2016:9060143.

24. Zhao X, Guan JL. Focal adhesion kinase and its signaling pathways in cell migration and angiogenesis. Adv Drug Deliv Rev 2011;63:610-5.

25. Liu B, Xu L, Yu X, et al. Genistein Inhibited Estradiol-Induced Vascular Endothelial Cell Injury by Downregulating the FAK/Focal Adhesion Pathway. Cell Physiol Biochem 2018;49:2277-92.

26. Chen L, Xie X, Zhang X, et al. The expression, regulation and adhesion function of a novel CD molecule, CD226, on human endothelial cells. Life Sci 2003;73:2373-82.

27. Bao H, Yao QP, Huang K, et al. Platelet-derived miR-142$3 \mathrm{p}$ induces apoptosis of endothelial cells in hypertension. Cell Mol Biol (Noisy-le-grand) 2017;63:3-9.

28. Blann AD, Nadar S, Lip GY. Pharmacological modulation of platelet function in hypertension. Hypertension $2003 ; 42: 1-7$.

29. Javerzat S, Franco M, Herbert J, et al. Correlating global gene regulation to angiogenesis in the developing chick extra-embryonic vascular system. PLoS One 2009;4:e7856.

30. Pitter B, Werner AC, Montanez E. Parvins Are Required for Endothelial Cell-Cell Junctions and Cell Polarity During Embryonic Blood Vessel Formation. Arterioscler Thromb Vasc Biol 2018;38:1147-58.

(English Language Editor: J. Jones) 
Supplementary

Table S1 qRT-PCR primer sequences

\begin{tabular}{lll}
\hline LncRNA or mRNA & Forward primer (5'-3') & Reverse primer (5'-3') \\
\hline ENST00000565493 & CAGTCCCTTGTGAATGTCCT & ACCCTTCCTCTTCTCCCTCTT \\
ENST00000606054 & GTCCCTGGCATTCCCGTGCTG & CCGGTGGATTCTCTCCGTTCC \\
ENST00000590604 & ATGAAGATGGGAAATTAACGC & GACAAAGAGGCTGTGCTGAAA \\
NONHSAT138949.2 & CAGAACGGCAACACCAAGCAA & TCCCACGCATAGTCGTGACCC \\
ENST00000566942 & CTGACATTTCTAATCTCCCTT & CTGTCCTCCACTAATCACAGA \\
ENST00000369385 & AAAGCCCAGAAGAAAGACGGCAAGA & TGGACCCGCTTCAGCACCTTG \\
NONHSAT217189.1 & GCACAGGTAAATGGTGGGAGT & CGCTGGATAGTAGGTAGGGAC \\
ENST00000540082 & CGAGGAGTGAGTTGAGCTTC & ACTTGGATTTGTCAGGTGGTC \\
CD226 & TAAATGGCTATTGGTTGCGTAT & CAAAGCTGGAAAGTTGAAAG \\
PARVB & TCAAGAAACCCAAGGCTCGTC & TGGAAAGGCACAGGGCACAGA \\
GAPDH & GCACCGTCAAGGCTGAGAAC & TGGTGAAGACGCCAGTGGA \\
B-actin & AAGGAGCCCCACGAGAAAAAT & ACCGAACTTGCATTGATTCCAG \\
\hline
\end{tabular}

(c) Annals of Translational Medicine. All rights reserved. 
Table S2 Go enrichment

\begin{tabular}{|c|c|c|c|c|c|c|c|c|c|}
\hline GO_ID & GO_term & TYPE & $\begin{array}{l}\text { target_gene } \\
\text { in_this_- } \\
\text { GO }\end{array}$ & $\begin{array}{l}\text { all_target_ } \\
\text { gene__in_ } \\
\text { all_GO }\end{array}$ & $\begin{array}{l}\text { all_gene_ } \\
\text { in_this_- } \\
\text { GO }\end{array}$ & $\begin{array}{c}\text { all_gene_ } \\
\text { in_all_ } \\
\text { GO }\end{array}$ & gene_list & Pvalue & Qvalue \\
\hline GO:0050839 & $\begin{array}{l}\text { cell adhesion } \\
\text { molecule binding }\end{array}$ & molecular_function & 4 & 43 & 198 & 21445 & ENSG00000150637 (CD226), ENSG00000135124 (P2RX4), ENSG00000259207 (ITGB3), ENSG00000150093 (ITGB1), & 0.000126273 & 0.192061381 \\
\hline GO:0019900 & kinase binding & molecular_function & 4 & 43 & 610 & 21445 & ENSG00000114302 (PRKAR2A), ENSG00000169756 (LIMS1), ENSG00000150093 (ITGB1), ENSG00000150637 (CD226), & 0.016991975 & 0.923028374 \\
\hline GO:0042802 & $\begin{array}{l}\text { identical protein } \\
\text { binding }\end{array}$ & molecular_function & 7 & 43 & 1268 & 21445 & $\begin{array}{l}\text { ENSG00000150637 (CD226), ENSG00000101457 (DNTTIP1), ENSG00000259207 (ITGB3), ENSG00000066427 (ATXN3), ENSG00000177119 (ANO6), } \\
\text { ENSG00000135124 (P2RX4), ENSG00000119917 (IFIT3), }\end{array}$ & 0.009510651 & 0.688842876 \\
\hline GO:0046983 & $\begin{array}{l}\text { protein } \\
\text { dimerization } \\
\text { activity }\end{array}$ & molecular_function & 6 & 43 & 1207 & 21445 & $\begin{array}{l}\text { ENSG00000177119 (ANO6), ENSG00000135124 (P2RX4), ENSG00000150093 (ITGB1), ENSG00000150637 (CD226), ENSG00000101457 (DNTTIP1), } \\
\text { ENSG00000061918 (GUCY1B3), }\end{array}$ & 0.024453622 & 1 \\
\hline GO:0019899 & enzyme binding & molecular_function & 7 & 43 & 1792 & 21445 & $\begin{array}{l}\text { ENSG00000114302 (PRKAR2A), ENSG00000169756 (LIMS1), ENSG00000150093 (ITGB1), ENSG00000147475 (ERLIN2), ENSG00000150637 (CD226), } \\
\text { ENSG00000259207 (ITGB3), ENSG00000066427 (ATXN3), }\end{array}$ & 0.061460033 & 1 \\
\hline GO:0005102 & receptor binding & molecular_function & 6 & 43 & 1628 & 21445 & $\begin{array}{l}\text { ENSG00000150093 (ITGB1), ENSG00000117899 (MESDC2), ENSG00000135124 (P2RX4), ENSG00000198721 (ECl2), ENSG00000259207 (ITGB3), } \\
\text { ENSG00000150637 (CD226), }\end{array}$ & 0.097893242 & 1 \\
\hline GO:0046914 & $\begin{array}{l}\text { transition metal ion } \\
\text { binding }\end{array}$ & molecular_function & 5 & 43 & 1579 & 21445 & ENSG00000256671 (LIMS3L), ENSG00000278129 (ZNF8), ENSG00000169756 (LIMS1), ENSG00000001630 (CYP51A1), ENSG00000135124 (P2RX4), & 0.204021337 & 1 \\
\hline GO:0005215 & transporter activity & molecular_function & 4 & 43 & 1341 & 21445 & ENSG00000177119 (ANO6), ENSG00000250021 (C15or(38-AP3S2), ENSG00000135124 (P2RX4), ENSG00000170144 (HNRNPA3), & 0.271865206 & 1 \\
\hline GO:0046872 & metal ion binding & molecular_function & 13 & 43 & 4408 & 21445 & $\begin{array}{l}\text { ENSG00000135482 (ZC3H1), ENSG00000150093 (ITGB1), ENSG00000163590 (PPM1L), ENSG00000061918 (GUCY1B3), ENSG000000278129 (ZNF8), } \\
\text { ENSG00000169756 (LIMS1), ENSG00000135124 (P2RX4), ENSG00000001630 (CYP51A1), ENSG00000167625 (ZNF526), ENSG00000256671 (LIMS3L), } \\
\text { ENSG00000198482 (ZNF808), ENSG00000172183 (ISG20), ENSG00000241404 (EGFL8), }\end{array}$ & 0.132599619 & 1 \\
\hline GO:0003723 & RNA binding & molecular_function & 5 & 43 & 1715 & 21445 & ENSG00000172183 (ISG20), ENSG00000119917 (IFIT3), ENSG00000049883 (PTCD2), ENSG00000135482 (ZC3H10), ENSG00000170144 (HNRNPA3), & 0.269727149 & 1 \\
\hline GO:0043169 & cation binding & molecular_function & 13 & 43 & 4496 & 21445 & $\begin{array}{l}\text { ENSG00000278129 (ZNF8), ENSG00000061918 (GUCY1B3), ENSG00000135124 (P2RX4), ENSG00000169756 (LIMS1), ENSG00000135482 (ZC3H10), } \\
\text { ENSG00000150093 (ITGB1), ENSG00000163590 (PPM1L), ENSG00000198482 (ZNF808), ENSG00000167625 (ZNF526), ENSG00000256671 (LIMS3L), } \\
\text { ENSG00000241404 (EGFL8), ENSG00000172183 (ISG20), ENSG0000001630 (CYP51A1), }\end{array}$ & 0.149832621 & 1 \\
\hline GO:0043167 & ion binding & molecular_function & 18 & 43 & 6572 & 21445 & $\begin{array}{l}\text { ENSG00000061918 (GUCY1B3), ENSG00000041353 (RAB27B), ENSG00000278129 (ZNF8), ENSG00000169756 (LIMS1), ENSG00000135124 (P2RX4), } \\
\text { ENSG00000135482 (ZC3H10), ENSG00000150093 (ITGB1), ENSG00000163590 (PPM1L), ENSG00000198721 (ECI2), ENSG00000107679 (PLEKHA1), } \\
\text { ENSG00000074935 (TUBE1), ENSG00000167625 (ZNF526), ENSG00000256671 (LIMSYL), ENSG00000198482 (ZNF808), ENSG00000172183 (ISG20), } \\
\text { ENSG00000241404 (EGFL), ENSG00000114302 (PRKAR2A), ENSG00000001630 (CYP51A1), }\end{array}$ & 0.15484274 & 1 \\
\hline GO:0097159 & $\begin{array}{l}\text { organic cyclic } \\
\text { compound binding }\end{array}$ & molecular_function & 17 & 43 & 6392 & 21445 & $\begin{array}{l}\text { ENSG00000135124 (P2RX4), ENSG00000278129 (ZNF8), ENSG00000066427 (ATXN3), ENSG00000041353 (RAB27B), ENSG00000061918 (GUCY1B3), } \\
\text { ENSG00000135482 (ZC3H10), ENSG00000172183 (ISG20), ENSG00000119917 (IFIT3), ENSG00000147475 (ERLIN2), ENSG00000101457 (DNTTIP1), } \\
\text { ENSG00000198482 (ZNF808), ENSG00000074935 (TUBE1), ENSG00000167625 (ZNF526), ENSG00000049883 (PTCD2), } \\
\text { ENSG00000001630 (CYP51A1), ENSG00000114302 (PRKAR2A), ENSG00000170144 (HNRNPA3), }\end{array}$ & 0.200137941 & 1 \\
\hline GO:0016787 & hydrolase activity & molecular_function & 7 & 43 & 2701 & 21445 & $\begin{array}{l}\text { ENSG00000163590 (PPM1L), ENSG00000155099 (TMEM55A), ENSG00000074935 (TUBE1), ENSG00000066427 (ATXN3), ENSG00000147003 } \\
\text { (TMEM27), ENSG00000041353 (RAB27B), ENSG00000172183 (ISG20), }\end{array}$ & 0.361361838 & 1 \\
\hline GO:0004872 & receptor activity & molecular_function & 5 & 43 & 1978 & 21445 & ENSG00000135124 (P2RX4), ENSG00000150093 (ITGB1), ENSG00000150637 (CD226), ENSG00000259207 (ITGB3), ENSG00000061918 (GUCY1B3), & 0.420143897 & 1 \\
\hline GO:0043168 & anion binding & molecular_function & 7 & 43 & 2837 & 21445 & $\begin{array}{l}\text { ENSG00000135124 (P2RX4), ENSG00000041353 (RAB27B), ENSG00000061918 (GUCY1B3), ENSG00000107679 (PLEKHA1), } \\
\text { ENSG00000074935 (TUBE1), ENSG00000114302 (PRKAR2A), ENSG00000198721 (ECI2), }\end{array}$ & 0.429593941 & 1 \\
\hline GO:0003824 & catalytic activity & molecular_function & 15 & 43 & 6133 & 21445 & $\begin{array}{l}\text { ENSG00000066427 (ATXN3), ENSG00000041353 (RAB27B), ENSG00000061918 (GUCY1B3), ENSG00000164181 (ELOVL7), ENSG00000198721 (ECL2), } \\
\text { ENSG00000163590 (PPM1L), ENSG00000074935 (TUBE1), ENSG00000155099 (TMEM55A), ENSG00000147003 (TMEM27), } \\
\text { ENSG00000172183 (ISG20), ENSG0000123427 (METTL21B), ENSG00000133997 (MED6), ENSG0000137364 (TPMT), ENSG00000259207 (ITGB3), } \\
\text { ENSG00000001630 (CYP51A1), }\end{array}$ & 0.356539494 & 1 \\
\hline GO:0036094 & $\begin{array}{l}\text { small molecule } \\
\text { binding }\end{array}$ & molecular_function & 7 & 43 & 2866 & 21445 & $\begin{array}{l}\text { ENSG00000135124 (P2RX4), ENSG00000147475 (ERLIN2), ENSG00000074935 (TUBE1), ENSG00000061918 (GUCY1B3), ENSG00000041353 } \\
\text { (RAB27B), ENSG00000114302 (PRKAR2A), ENSG00000170144 (HNRNPA3), }\end{array}$ & 0.4447432 & 1 \\
\hline GO:0000166 & nucleotide binding & molecular_function & 6 & 43 & 2555 & 21445 & $\begin{array}{l}\text { ENSG00000135124 (P2RX4), ENSG00000061918 (GUCY1B3), ENSG00000041353 (RAB27B), ENSG00000074935 (TUBE1), ENSG00000114302 } \\
\text { (PRKAR2A), ENSG00000170144 (HNRNPA3), }\end{array}$ & 0.512921749 & 1 \\
\hline GO:0003676 & $\begin{array}{l}\text { nucleic acid } \\
\text { binding }\end{array}$ & molecular_function & 10 & 43 & 4265 & 21445 & $\begin{array}{l}\text { ENSG00000119917 (IFIT3), ENSG00000172183 (ISG20), ENSG00000167625 (ZNF526), ENSG00000198482 (ZNF808), ENSG00000101457 (DNTTIP1), } \\
\text { ENSG00000049883 (PTCD2), ENSG00000170144 (HNRNAA), ENSG00000066427 (ATXN3), ENSG00000278129 (ZNF8), ENSG00000135482 (ZC3H10), }\end{array}$ & 0.48555041 & 1 \\
\hline GO:0005515 & protein binding & molecular_function & 29 & 43 & 14009 & 21445 & 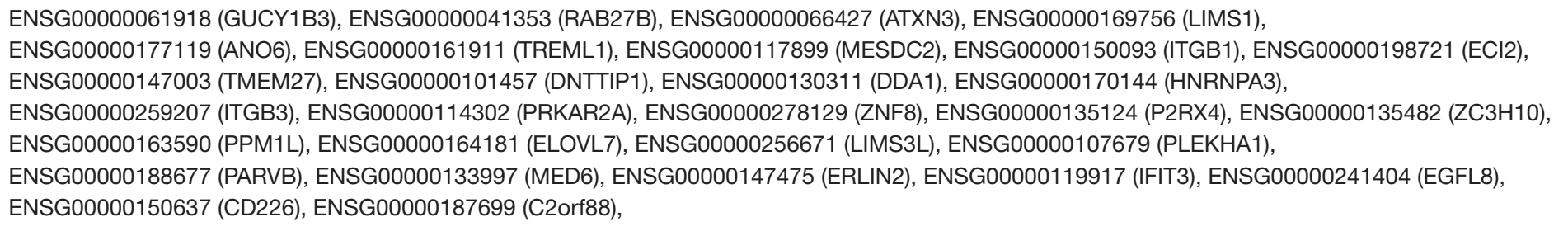 & 0.768267171 & 1 \\
\hline GO:0005488 & binding & molecular_function & 35 & 43 & 17182 & 21445 & 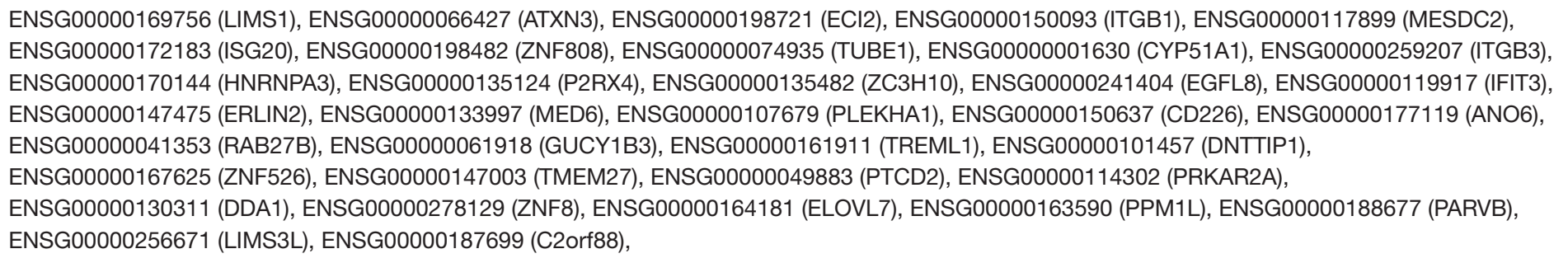 & 0.837150357 & 1 \\
\hline GO:0035639 & $\begin{array}{l}\text { purine } \\
\text { ribonucleoside } \\
\text { triphosphate } \\
\text { binding }\end{array}$ & molecular_function & 4 & 43 & 1985 & 21445 & ENSG00000041353 (RAB27B), ENSG00000061918 (GUCY1B3), ENSG00000074935 (TUBE1), ENSG00000135124 (P2RX4), & 0.735372916 & 1 \\
\hline GO:0001883 & $\begin{array}{l}\text { purine nucleoside } \\
\text { binding }\end{array}$ & molecular_function & 4 & 43 & 1998 & 21445 & ENSG00000135124 (P2RX4), ENSG00000061918 (GUCY1B3), ENSG00000041353 (RAB27B), ENSG00000074935 (TUBE1), & 1 & 1 \\
\hline GO:0032549 & $\begin{array}{l}\text { ribonucleoside } \\
\text { binding }\end{array}$ & molecular_function & 4 & 43 & 1998 & 21445 & ENSG00000074935 (TUBE1), ENSG00000041353 (RAB27B), ENSG00000061918 (GUCY1B3), ENSG00000135124 (P2RX4), & 1 & 1 \\
\hline GO:0001882 & nucleoside binding & molecular_function & 4 & 43 & 2005 & 21445 & ENSG00000135124 (P2RX4), ENSG00000074935 (TUBE1), ENSG00000041353 (RAB27B), ENSG00000061918 (GUCY1B3), & 1 & 1 \\
\hline GO:0003677 & DNA binding & molecular_function & 5 & 43 & 2569 & 21445 & ENSG00000066427 (ATXN3), ENSG00000167625 (ZNF526), ENSG00000198482 (ZNF808), ENSG00000278129 (ZNF8), ENSG00000101457 (DNTTIP1), & 1 & 1 \\
\hline GO:0016740 & transferase activity & molecular_function & 4 & 43 & 2361 & 21445 & ENSG00000164181 (ELOVL7), ENSG00000137364 (TPMT), ENSG00000133997 (MED6), ENSG00000123427 (METTL21B) & 1 & 1 \\
\hline
\end{tabular}




\begin{tabular}{|c|c|c|c|c|c|c|c|c|}
\hline pathway_ID & PATHWAY_DES & $\begin{array}{l}\text { target_gene_in_this_ } \\
\text { pathway }\end{array}$ & $\begin{array}{c}\text { target_gene_in_all_ } \\
\text { pathway }\end{array}$ & $\begin{array}{c}\text { all_gene_in_this_ } \\
\text { pathway }\end{array}$ & $\begin{array}{c}\text { all_gene_in_all__ } \\
\text { pathway }\end{array}$ & all_gene_list & Pvalue & Qvalue \\
\hline hsa05412 & $\begin{array}{l}\text { Arrhythmogenic right ventricular } \\
\text { cardiomyopathy (ARVC) }\end{array}$ & 2 & 15 & 74 & 6914 & ENSG00000150093 (ITGB1), ENSG00000259207 (ITGB3), & 0.001720314 & 0.041287533 \\
\hline hsa04611 & Platelet activation & 3 & 15 & 122 & 6914 & ENSG00000150093 (ITGB1), ENSG00000061918 (GUCY1B3), ENSG00000259207 (ITGB3), & 0.000568765 & 0.027300739 \\
\hline hsa04512 & ECM-receptor interaction & 2 & 15 & 82 & 6914 & ENSG00000150093 (ITGB1), ENSG00000259207 (ITGB3), & 0.002303211 & 0.036851376 \\
\hline hsa05410 & Hypertrophic cardiomyopathy (HCM) & 2 & 15 & 83 & 6914 & ENSG00000150093 (ITGB1), ENSG00000259207 (ITGB3), & 0.002383691 & 0.028604288 \\
\hline hsa05414 & Dilated cardiomyopathy & 2 & 15 & 89 & 6914 & ENSG00000150093 (ITGB1), ENSG00000259207 (ITGB3), & 0.002903562 & 0.027874194 \\
\hline hsa04510 & Focal adhesion & 3 & 15 & 202 & 6914 & ENSG00000150093 (ITGB1), ENSG00000188677 (PARVB), ENSG00000259207 (ITGB3), & 0.003575027 & 0.028600218 \\
\hline hsa04145 & Phagosome & 2 & 15 & 153 & 6914 & ENSG00000150093 (ITGB1), ENSG00000259207 (ITGB3), & 0.012980299 & 0.077881792 \\
\hline hsa05205 & Proteoglycans in cancer & 2 & 15 & 203 & 6914 & ENSG00000150093 (ITGB1), ENSG00000259207 (ITGB3), & 0.027474421 & 0.146530247 \\
\hline hsa04015 & Rap1 signaling pathway & 2 & 15 & 211 & 6914 & ENSG00000150093 (ITGB1), ENSG00000259207 (ITGB3), & 0.030373543 & 0.145793004 \\
\hline hsa04810 & Regulation of actin cytoskeleton & 2 & 15 & 214 & 6914 & ENSG00000150093 (ITGB1), ENSG00000259207 (ITGB3), & 0.031502562 & 0.137465724 \\
\hline hsa04151 & PI3K-Akt signaling pathway & 2 & 15 & 340 & 6914 & ENSG00000150093 (ITGB1), ENSG00000259207 (ITGB3), & 0.099191119 & 0.396764476 \\
\hline hsa05130 & Pathogenic Escherichia coli infection & 1 & 15 & 55 & 6914 & ENSG00000150093 (ITGB1), & 1 & 1 \\
\hline hsa04910 & Insulin signaling pathway & 1 & 15 & 139 & 6914 & ENSG00000114302 (PRKAR2A), & 1 & 1 \\
\hline hsa05145 & Toxoplasmosis & 1 & 15 & 118 & 6914 & ENSG00000150093 (ITGB1), & 1 & 1 \\
\hline hsa04146 & Peroxisome & 1 & 15 & 83 & 6914 & ENSG00000198721 (ECI2), & 1 & 1 \\
\hline hsa05200 & Pathways in cancer & 1 & 15 & 397 & 6914 & ENSG00000150093 (ITGB1), & 1 & 1 \\
\hline hsa05100 & Bacterial invasion of epithelial cells & 1 & 15 & 78 & 6914 & ENSG00000150093 (ITGB1), & 1 & 1 \\
\hline hsa04730 & Long-term depression & 1 & 15 & 60 & 6914 & ENSG00000061918 (GUCY1B3), & 1 & 1 \\
\hline hsa04713 & Circadian entrainment & 1 & 15 & 95 & 6914 & ENSG00000061918 (GUCY1B3), & 1 & 1 \\
\hline hsa05222 & Small cell lung cancer & 1 & 15 & 86 & 6914 & ENSG00000150093 (ITGB1), & 1 & 1 \\
\hline hsa04670 & Leukocyte transendothelial migration & 1 & 15 & 118 & 6914 & ENSG00000150093 (ITGB1), & 1 & 1 \\
\hline hsa00062 & Fatty acid elongation & 1 & 15 & 25 & 6914 & ENSG00000164181 (ELOVL7), & 1 & 1 \\
\hline hsa03040 & Spliceosome & 1 & 15 & 134 & 6914 & ENSG00000170144 (HNRNPA3), & 1 & 1 \\
\hline hsa04020 & Calcium signaling pathway & 1 & 15 & 179 & 6914 & ENSG00000135124 (P2RX4), & 1 & 1 \\
\hline hsa01100 & Metabolic pathways & 1 & 15 & 1238 & 6914 & ENSG00000001630 (CYP51A1), & 1 & 1 \\
\hline hsa04022 & cGMP-PKG signaling pathway & 1 & 15 & 167 & 6914 & ENSG00000061918 (GUCY1B3), & 1 & 1 \\
\hline hsa04919 & Thyroid hormone signaling pathway & 1 & 15 & 118 & 6914 & ENSG00000259207 (ITGB3), & 1 & 1 \\
\hline hsa04972 & Pancreatic secretion & 1 & 15 & 96 & 6914 & ENSG00000041353 (RAB27B), & 1 & 1 \\
\hline hsa00100 & Steroid biosynthesis & 1 & 15 & 20 & 6914 & ENSG00000001630 (CYP51A1), & 1 & 1 \\
\hline hsa05133 & Pertussis & 1 & 15 & 75 & 6914 & ENSG00000150093 (ITGB1), & 1 & 1 \\
\hline hsa04540 & Gap junction & 1 & 15 & 88 & 6914 & ENSG00000061918 (GUCY1B3), & 1 & 1 \\
\hline hsa05206 & MicroRNAs in cancer & 1 & 15 & 256 & 6914 & ENSG00000259207 (ITGB3), & 1 & 1 \\
\hline hsa04924 & Renin secretion & 1 & 15 & 64 & 6914 & ENSG00000061918 (GUCY1B3), & 1 & 1 \\
\hline hsa04640 & Hematopoietic cell lineage & 1 & 15 & 87 & 6914 & ENSG00000259207 (ITGB3), & 1 & 1 \\
\hline hsa04360 & Axon guidance & 1 & 15 & 173 & 6914 & ENSG00000150093 (ITGB1), & 1 & 1 \\
\hline hsa00983 & Drug metabolism - other enzymes & 1 & 15 & 46 & 6914 & ENSG00000137364 (TPMT), & 1 & 1 \\
\hline hsa05140 & Leishmaniasis & 1 & 15 & 72 & 6914 & ENSG00000150093 (ITGB1), & 1 & 1 \\
\hline hsa05131 & Shigellosis & 1 & 15 & 65 & 6914 & ENSG00000150093 (ITGB1), & 1 & 1 \\
\hline hsa04141 & Protein processing in endoplasmic reticulum & 1 & 15 & 169 & 6914 & ENSG00000066427 (ATXN3), & 1 & 1 \\
\hline hsa04270 & Vascular smooth muscle contraction & 1 & 15 & 120 & 6914 & ENSG00000061918 (GUCY1B3), & 1 & 1 \\
\hline hsa04080 & Neuroactive ligand-receptor interaction & 1 & 15 & 277 & 6914 & ENSG00000135124 (P2RX4), & 1 & 1 \\
\hline hsa04921 & Oxytocin signaling pathway & 1 & 15 & 158 & 6914 & ENSG00000061918 (GUCY1B3), & 1 & 1 \\
\hline hsa04070 & Phosphatidylinositol signaling system & 1 & 15 & 98 & 6914 & ENSG00000155099 (TMEM55A), & 1 & 1 \\
\hline
\end{tabular}

